\title{
Campo elétrico do capacitor de placas retangulares finito
}

\author{
Helder, H. Ch. S. ${ }^{1}$; Kariston, A. P. ${ }^{1}$; Lucas, N. ${ }^{1}$; Kesley, S. ${ }^{1}$; Eloi Silva ${ }^{2}$ \\ 1 Núcleo Básico de Engenharia, Faculdade Centro Leste, Rodovia ES 010, km 6, Manguinhos - Serra - ES \\ 2 Departamento de Química, Universidade Federal do Espírito Santo, Av. Fernando Ferrari 514, Goiabeiras - Vitória - \\ ES - 29075-910
}

\begin{abstract}
Resumo
Este trabalho apresenta expressões exatas para as componentes dos campos elétricos de um capacitor de placas retangulares finitas, para qualquer ponto dentro e fora das placas, os resultados computacionais utilizando o programa Mathematica para os campos elétricos mostram a influência dos efeitos de borda sobre a distribuição do campo elétrico.

Keywords (Palavras chaves): campo elétrico, dipolo elétrico, capacitor, efeito de borda.
\end{abstract}

\section{Introdução}

O capacitor de placas planas infinitas é um tema muito conhecido dos livros de física básica [1], no qual o campo elétrico é constante dentro das placa $\vec{E}=\frac{\sigma}{\varepsilon} \hat{n}$ e zero fora delas, sendo $\sigma$ a densidade superficial de cargas nas placas. Pode ser provado, que o mesmo campo seja obtido para um capacitor de placas circulares com o raio da placa $R>>z$, onde $z$ é a distância de separação entre as placas. No entanto, o caso de um capacitor onde a distância entre as placas é da mesma ordem de grandeza que as dimensões das placas tem resistido ao cálculo analítico exato, conhecendo-se apenas métodos numéricos baseados em diferenças finitas a fim de resolver a equação de Poisson correspondente [2,3]. Apresentamos os resultados computacionais de um cálculo analítico exato dos campos elétricos de um capacitor de placas planas retangulares cujas dimensões são comparáveis com a distância de separação entre as placas, um resultado que não era conhecido na literatura. Nossos cálculos estão baseados em princípios básicos do campo elétrico de um elemento diferencial de carga e nas técnicas de integração. Na seção 2, descrevemos o procedimento do cálculo do campo elétrico e na seção 3 apresentamos os resultados computacionais dos mesmos. $\mathrm{Na}$ seção 4 discutimos os resultados e concluímos com alguns comentários.

\section{Campo elétrico de um capacitor de placas retangulares}

A Figura 1 ilustra o método do cálculo do campo elétrico num ponto arbitrário $P(x, y, z)$. Escolhemos elementos de superfície nas placas $d S^{\prime}=d x^{\prime} d y^{\prime}$ nas quais está distribuída cargas elementares $\mathrm{dQ}^{\prime}=\sigma \mathrm{d} \mathrm{S}^{\prime}$. Ambas as cargas se encontram na mesma posição $\left(x^{\prime}, y^{\prime}\right)$ no sistema de coordenadas associadas as placas. Com isto em mente, escrevemos as componentes dos campos elétricos:

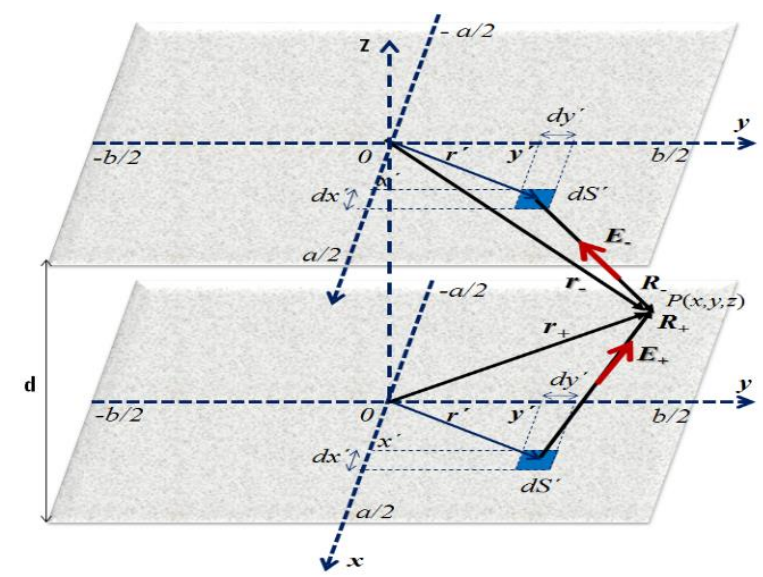

Figura 1: Duas placas condutoras com dimensões $a \times b$ e densidade de cargas $\sigma$ constante, a placa inferior contendo cargas positivas e a superior negativas. 


$$
\begin{aligned}
& E_{x}=k \sigma \int_{-b / 2}^{\infty / 2} d y^{\sigma} \int_{-a / 2}^{a / 2}\left(\frac{1}{\left((x-x)^{2}+(y-y)^{2}+z^{2}\right)^{a / 2}}\right. \\
& -\frac{1}{\left.\left((x-x)^{2}+(y-y)^{2}+(d-z)^{2}\right)^{3 / 2}\right)\left(x-x^{\prime}\right) d x^{\prime}} \\
& E_{y}=k \sigma \int_{-b / 2}^{o / 2} d y^{\sigma} \int_{-a / 2}^{a / 2}\left(\frac{1}{\left((x-x)^{2}+(y-y)^{2}+z^{2}\right)^{2 / 2}}\right. \\
& \left.-\frac{1}{\left((x-x)^{2}+(y-y)^{2}+(d-z)^{2}\right)^{3 / 2}}\right)\left(y-y^{2}\right) d x^{x} \\
& E_{z}=k \sigma \int_{-b / 2}^{b_{j} / 2} d y^{\prime} \int_{-a / 2}^{a / 2}\left(\frac{z}{\left((x-x)^{2}+(y-y)^{2}+z^{2}\right)^{a / 2}}\right. \\
& \left.+\frac{d-z}{\left((x-x)^{2}+(y-y)^{2}+(d-z)^{2}\right)^{3 / 2}}\right) d x^{2}
\end{aligned}
$$

onde $o$ fator $k=1 /(4 \pi \varepsilon 0)$.

\section{Resultados computacionais}

O cálculo exato das integrais (1) - (3) foram obtidas com ajuda do Mathematica 9.0 [4,5], e as expressões são muito longas para serem escritas aqui. Em vez disso, são mostrados os gráficos para $a=1, b=2$ e $d=$ 1 , e os campos foram calculados como $E_{x} /(k \sigma), E_{y} /(k \sigma)$ e $E_{z} /(k \sigma)$.
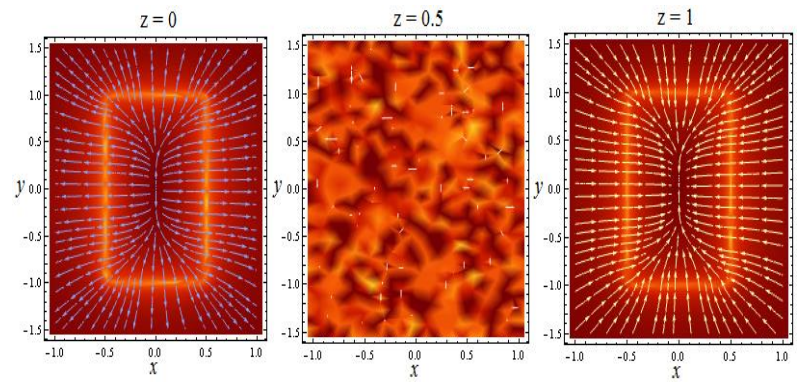

Figura 2: Gráficos das componentes $E_{x}$ vs $E_{y}$ do campo elétrico para $z=0,0.5$ e 1 respectivamente.
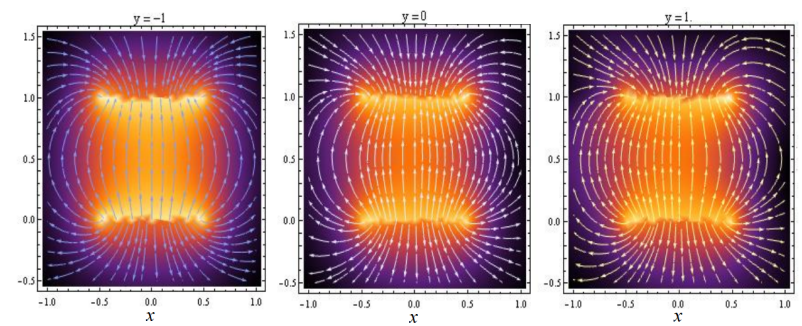

Figura 3: Gráficos das componentes $E_{x}$ vs $E_{z}$ do campo elétrico para $y=-1,0$ e 1 respectivamente.
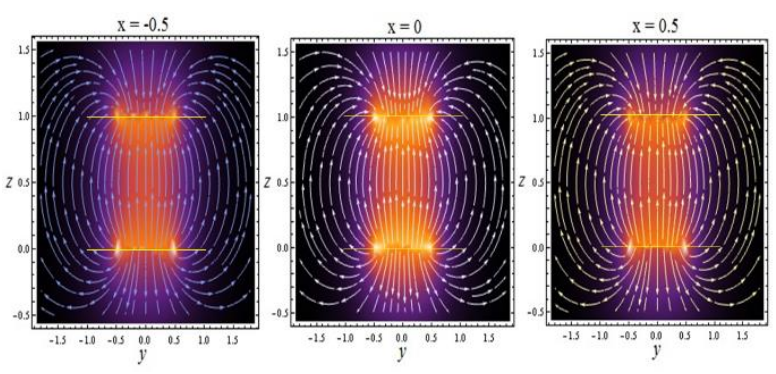

Figura 4: Gráficos das componentes $E_{y}$ vs $E_{z}$ do campo elétrico para $x=-0.5,0$ e 0.5 respectivamente.

\section{Análise dos resultados}

A Figura 2 mostra que as componentes do campo no plano $X Y\left(E_{x}=-\partial \varphi / \partial x, E_{y}=-\partial \varphi / \partial y\right)$ são diferentes de zero em todos os pontos do espaço exceto no plano intermédio $z=0.5$. Isto é facilmente compreensível dado que todos os pontos que formam o plano equipotencial $\varphi(x, y, 0.5)=0$. Deste modo, observam-se os efeitos de borda do campo elétrico nos planos $y=-1,0,1$, onde a componente $x$ do campo começa a se manifestar nas bordas das placas e dentro das placas o campo é essencialmente ao longo do eixo $z$. $\mathrm{Na}$ Figura 4, mostra-se as componentes $\mathrm{E}_{\mathrm{y}}$ vs $\mathrm{E}_{\mathrm{z}}$. Outra vez os efeitos de borda são mais evidentes quando olhados nos planos $x=-0.5,0,0.5$.

\section{Agradecimentos}

Agradecemos a FAPES pelo apoio financeiro e ao CCE-UFES por nos permitir usar seus recursos computacionais para desenvolver esta pesquisa e ao Núcleo Básico da UCL por toda ajuda fornecida.

\section{Referências}

[1] TIPLER, P. A.; MOSCA, G. Physic for Scientists and Engineers. W. H. Freeman and Company, 2008.

[2]. PARKER, G. W. Computers in Physics, v.5, p. 534540, 1991.

[3] BUENO-BARRACHINA, J. M; CAÑAS-PAÑUELAS, C. S; CATALAN-IZQUIERDO, S, Journal of Energy and Power Engineering v5, p. 373-378, 2011.

[4] Disponível em < www.Wolfram.com> acesso em: 02 de Março de 2014.

[5] TROTT, M, THE MATHEMATICA GUIDEBOOK for Symbolics, Springer, 2006. 